CRYSTALLOGRAPHIC COMMUNICATIONS

ISSN 2056-9890

\section{Crystal structure and Hirshfeld surface analysis of 2-hydroxy-7-methoxy-1,8-bis(2,4,6-trichlorobenzo- yl)naphthalene}

\author{
Toyokazu Muto, ${ }^{a}$ Kikuko lida, ${ }^{a}$ Keiichi Noguchi, ${ }^{b}$ Noriyuki Yonezawa ${ }^{a}$ and Akiko \\ Okamoto $^{\mathrm{a} *}$
}

\begin{abstract}
a Department of Organic and Polymer Materials Chemistry, Tokyo University of Agriculture and Technology, Koganei, Tokyo 184-8588, Japan, and ${ }^{\mathbf{b}}$ Instrumentation Analysis Center, Tokyo University of Agriculture and Technology, Koganei, Tokyo 184-8588, Japan. *Correspondence e-mail: aokamoto@cc.tuat.ac.jp
\end{abstract}

Edited by $\mathrm{H}$. Stoeckli-Evans, University of Neuchâtel, Switzerland

Keywords: crystal structure; non-coplanar accumulated aromatic rings structure; twisted aroyl group; intramolecular $\mathrm{O}-\mathrm{H}$... O hydrogen bond; short contacts involving chloro group; herringbone pattern; Hirshfeld surface analysis; two-dimensional fingerprint plots.

CCDC reference: 1950725

Supporting information: this article has supporting information at journals.iucr.org/e

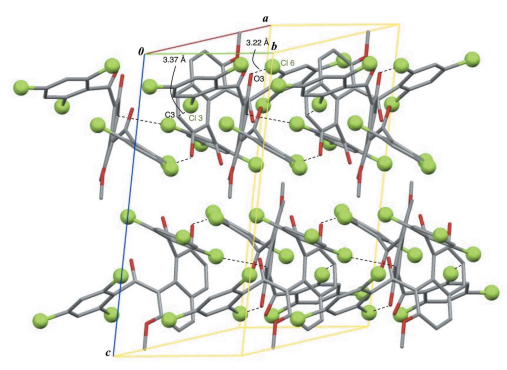

In the title compound, $\mathrm{C}_{25} \mathrm{H}_{12} \mathrm{Cl}_{6} \mathrm{O}_{4}$, the two carbonyl groups are oriented in a same direction with respect to the naphthalene ring system and are situated roughly parallel to each other, while the two 2,4,6-trichlorobenzene rings are orientated in opposite directions with respect to the naphthalene ring system: the carbonyl $\mathrm{C}-(\mathrm{C}=\mathrm{O})-\mathrm{C}$ planes subtend dihedral angles of $45.54(15)$ and $30.02(15)^{\circ}$ to the naphthalene ring system are. The dihedral angles formed by the carbonyl groups and the benzene rings show larger differences, the $\mathrm{C}=\mathrm{O}$ vectors being inclined to the benzene rings by 46.39 (16) and $79.78(16)^{\circ}$. An intramolecular $\mathrm{O}-\mathrm{H} \cdots \mathrm{O}=\mathrm{C}$ hydrogen bond forms an $S(6)$ ring motif. In the crystal, no effective intermolecular hydrogen bonds are found; instead, $\mathrm{O} \cdots \mathrm{Cl}$ and $\mathrm{C} \cdots \mathrm{Cl}$ close contacts are observed along the $2_{1}$ helical-axis direction. The Hirshfeld surface analysis reveals several weak interactions, the major contributor being $\mathrm{Cl} \cdots \mathrm{H} / \mathrm{H} \cdots \mathrm{Cl}$ contacts.

\section{Chemical context}

$o$-Hydroxyaryl ketones are generally recognized to be important precursors in the preparation of valuable products such as drugs, cosmetics, dyes and pesticides (Choy \& Kwong, 2013; Naeimi et al., 2014; Nimnual et al., 2015). The preparation methods reported include, for example, Fries rearrangement of phenolic esters (Murashige et al., 2011), acylation of benzoquinone and derivatives (Schiel et al., 2001), coupling reactions of nitriles with boronic acids (Zhou \& Larock, 2004), direct $\mathrm{C}-\mathrm{H}$ bond arylation of 2-hydroxybenzaldehydes (Lee \& Yi, 2015; Weng et al., 2010), and microwave-assisted direct benzoylation of phenols under solvent-free or ionic liquid conditions (Tran et al., 2017). The neighbouring carbonyl and hydroxy groups contribute to the regio- and chemoselectivities in these reactions. Conformational studies of hydroxyaryl ketones in the solid state and in solution have attracted considerable interest (Siskos et al., 2015; Nonhebel, 1968). Since the discovery of an effective method for diaroylation at the 1,8(peri)-positions of the naphthalene ring core and the related reactions (Okamoto \& Yonezawa, 2009; Okamoto et al., 2011; Okamoto, Mitsui et al., 2012), we have reported on the spatial organization of 1,8-diaroylated naphthalenes and homologous compounds in both the solid state and solution (Okamoto, Watanabe et al., 2012; Yoshiwaka et al., 2015; Okamoto et al., 2015; Ohisa et al., 2018). In the crystal structures of these compounds, which have non-coplanar accumulated aromatic rings, molecules are arranged by weak 
intermolecular interactions, such as non-classical hydrogen bonds and van der Waals interactions. Thus, the accumulation structures of 1,8-diaroylated naphthalenes are drastically changed by simple molecular modifications. Herein, we report on the crystal structure and Hirshfeld surface analysis of the title hydroxyaryl ketone, 2-hydroxy-7-methoxy-1,8-bis(2,4,6trichlorobenzoyl)naphthalene.

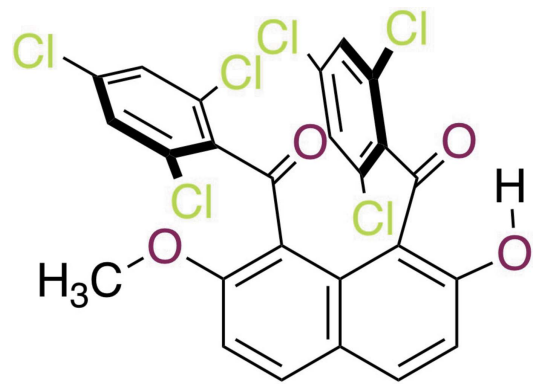

\section{Structural commentary}

The molecular structure of the title compound is shown in Fig. 1. This compound consists of a naphthalene ring core with two 2,4,6-trichlorobenzoyl groups at the 1,8-positions, a hydroxy group at the 2-position, and a methoxy group at the 7-position of the naphthalene ring system, affording an unsymmetrical molecular structure.

Analogous aroylated unsymmetrical naphthalene compounds, for example, 1,8-bis(4-chlorobenzoyl)-2-hydroxy7-methoxynaphthalene (Mitsui, Nagasawa, Noguchi et al., 2010) and 1,8-bis(4-chlorobenzoyl)-2,7-dimethoxynaphthalene (Nakaema et al., 2007), have two aroyl groups at the 1,8positions of the naphthalene ring system. The two 4-chlorobenzoyl groups have the same orientation with respect to the

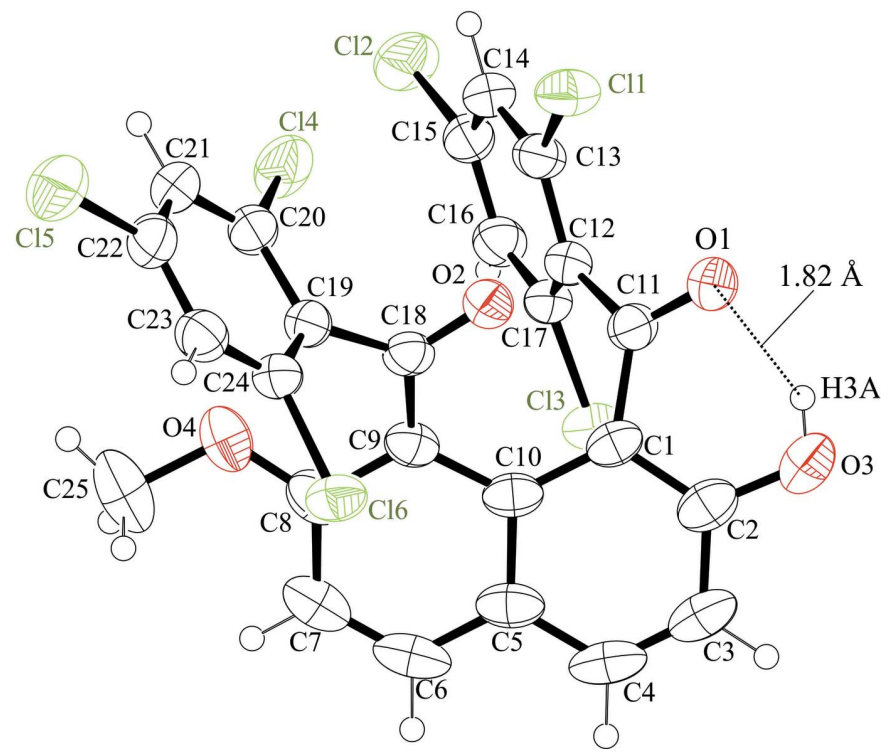

Figure 1

The molecular structure of the title compound, with atom labelling. Displacement ellipsoids are drawn at the $50 \%$ probability level.
Table 1

Hydrogen-bond geometry $\left(\AA,^{\circ}\right)$.

\begin{tabular}{lllll}
\hline$D-\mathrm{H} \cdots A$ & $D-\mathrm{H}$ & $\mathrm{H} \cdots A$ & $D \cdots A$ & $D-\mathrm{H} \cdots A$ \\
\hline $\mathrm{O} 3-\mathrm{H} 3 A \cdots \mathrm{O} 1$ & 0.84 & 1.82 & $2.551(3)$ & 145 \\
\hline
\end{tabular}

naphthalene ring core in 1,8-bis(4-chlorobenzoyl)-2-hydroxy7-methoxynaphthalene, while they are in opposite directions in 1,8-bis(4-chlorobenzoyl)-2,7-dimethoxynaphthalene. In contrast, in the title compound the carbonyl groups and the benzene rings of the 2,4,6-trichlorobenzoyl groups are located in distinct orientations with respect to the naphthalene ring plane: the two carbonyl groups are oriented in the same direction and are located roughly parallel to the naphthalene ring, whereas the two 2,4,6-trichlorobenzene rings are twisted away in opposite directions (Fig. 2). The dihedral angles of the carbonyl $\mathrm{C}-(\mathrm{C}=\mathrm{O})-\mathrm{C}$ plane $[\mathrm{C} 1-(\mathrm{C} 11=\mathrm{O} 1)-\mathrm{C} 12$ and $\mathrm{C} 9-(\mathrm{C} 18=\mathrm{O} 2)-\mathrm{C} 19]$ and the naphthalene ring are $45.54(15)$ and $30.02(15)^{\circ}$, respectively. The carbonyl C$(\mathrm{C}=\mathrm{O})-\mathrm{C}$ plane and the 2,4,6-trichlorobenzene ring in the 8-position of the naphthalene ring forms a larger dihedral angle than that in 1-position [C19-C24 ring and C12-C17 ring], $79.78(16)^{\circ}$ versus $46.39(16)^{\circ}$. The two carbonyl $\mathrm{C}-$ $(\mathrm{C}=\mathrm{O})-\mathrm{C}$ planes make a large dihedral angle, $73.68(19)^{\circ}$. Furthermore, the naphthalene ring plane is somewhat distorted, the $\mathrm{C} 6-\mathrm{C} 5-\mathrm{C} 10-\mathrm{C} 9$ and $\mathrm{C} 4-\mathrm{C} 5-\mathrm{C} 10-\mathrm{C} 1$ torsion angles being 10.2 (4) and $6.1(5)^{\circ}$, respectively.

The intramolecular $\mathrm{O}-\mathrm{H} \cdots \mathrm{O}=\mathrm{C}$ hydrogen bond forms a six-membered $S(6)$ ring motif $(\mathrm{O} 3-\mathrm{H} 3 A \cdots \mathrm{O} 1$; Figs. 1 and 2 , Table 1). In addition, one chloro atom of the trichlorobenzoyl group at the 1-position of the naphthalene ring system makes two short intramolecular $\mathrm{Cl} \cdots \mathrm{O}=\mathrm{C}$ contacts $[\mathrm{Cl} 1 \cdots \mathrm{O} 1=$ 3.018 (2) $\AA$ and $\mathrm{Cl} 1 \cdots \mathrm{O} 2=2.969$ (2) $\AA$ ] . 1-Aroyl-2-hydroxynaphthalene homologues often form intramolecular $\mathrm{O}-$ $\mathrm{H} \cdots \mathrm{O}=\mathrm{C}$ hydrogen bonds whether the second aroyl group is present or not, e.g., 1-benzoyl-2-hydroxy-7-methoxynaphthalene (Nagasawa, Mitsui, Kato et al., 2010), 2-hydroxy7-methoxy-1-(4-methylbenzoyl)naphthalene (Nagasawa, Mitsui, Okamoto et al., 2010), 1-(4-chlorobenzoyl)-2-hydroxy7-methoxynaphthalene (Mitsui et al., 2008) and 1,8-bis(4-
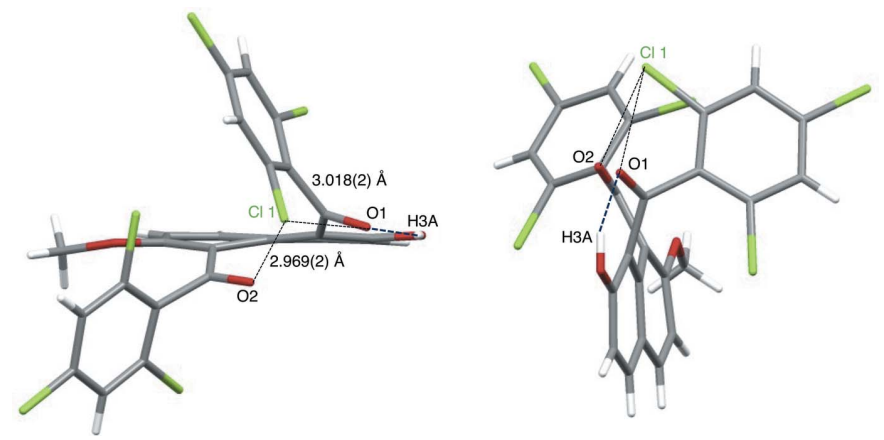

Figure 2

View of the title compound showing the intramolecular contacts; top view (left) and side view (right). 


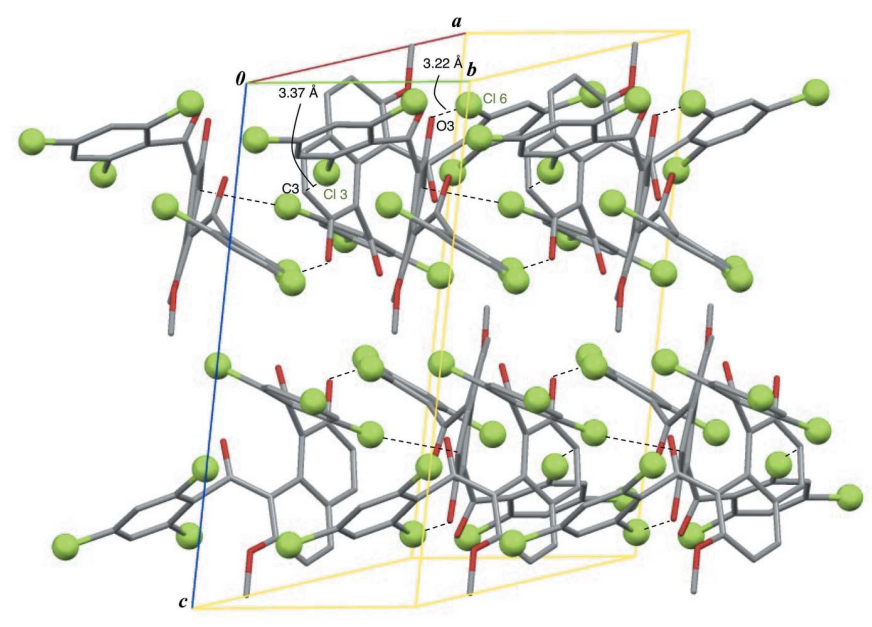

Figure 3

A view of the crystal packing of the title compound, showing the $\mathrm{Cl} \cdots \mathrm{O}$ and $\mathrm{Cl} \cdots \mathrm{C}$ short contacts. $\mathrm{H}$ atoms have been omitted for clarity.

chlorobenzoyl)-2-hydroxy-7-methoxynaphthalene (Mitsui, Nagasawa, Noguchi et al., 2010). The 2,4,6-trisubstituents in the benzene ring tend to bring about intramolecular short contacts involving the carbonyl oxygen atom: intramolecular $\mathrm{C}-\mathrm{H} \cdots \mathrm{O}=\mathrm{C}$ hydrogen bonds are observed in 2,7-dimethoxy-1,8-bis(2,4,6-trimethylbenzoyl)naphthalene (Muto et al., $2012 a$ ) and 1-(4-chlorobenzoyl)-2,7-dimethoxy-8-(2,4,6-trimethylbenzoyl)naphthalene (Muto et al., 2012b).

\section{Supramolecular features}

In the crystal, $2_{1}$ helical molecular assemblies are observed along the $b$-axis direction (Fig. 3). The chloro groups in the assemblies are aligned in a herringbone pattern. There are no

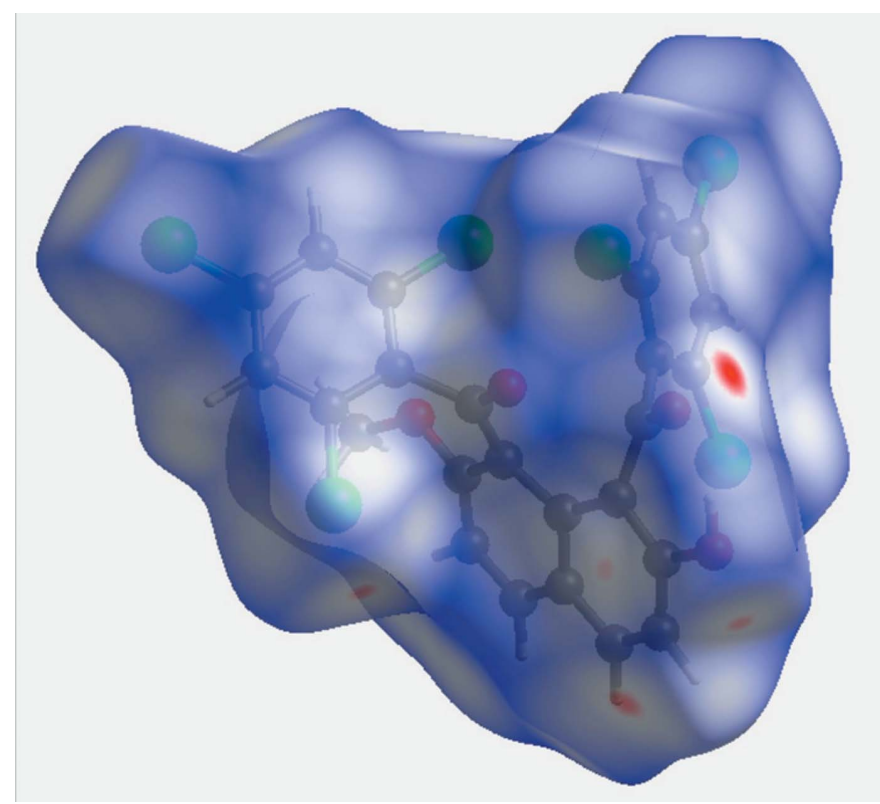

Figure 4

The Hirshfeld surface of the title compound mapped over $d_{\text {norm }}$, in the range -0.0895 to 1.1549 a.u. effective hydrogen bonds, instead, two kinds of short contacts involving chlorine atoms are observed; Cl6 . OO $3^{\mathrm{i}}$ [3.224 (3) A] and $\mathrm{Cl} 3 \cdots \mathrm{C} 3^{\mathrm{i}}\left[3.370\right.$ (3) $\AA$ ] , symmetry code: (i) $-x+1, y+\frac{1}{2}$, $\frac{1}{2}-z$ (Fig. 3).

\section{Hirshfeld surface analysis and two-dimensional fingerprint plots}

The Hirshfeld surface analysis (Spackman \& Jayatilaka, 2009) and the associated two-dimensional fingerprint plots (McKinnon et al., 2007) were performed with CrystalExplorer17 (Turner et al., 2017). The Hirshfeld surfaces are colour-mapped with the normalized contact distance, $d_{\text {norm }}$, from red (distances shorter than the sum of the van der Waals radii) through white to blue (distances longer than the sum of the van der Waals radii). The Hirshfeld surface of the title compound mapped over $d_{\text {norm }}$ in the range -0.0895 to 1.1549 a.u., is shown in Fig. 4. The red points represent close contacts and negative $d_{\text {norm }}$ values on the surface. The largest red point corresponds to the short contact of 3.078 (3) $\AA$ involving the carbonyl $\mathrm{O}$ atom, $\mathrm{O} 1$, and carbon atom $\mathrm{C} 23^{\mathrm{i}}$ [symmetry code: (i) $\left.x,-y+\frac{3}{2}, z-\frac{1}{2}\right]$, while the other red points around the naphthalene ring indicate short $\mathrm{Cl} \cdots \mathrm{H}$ interactions.

The two-dimensional fingerprint plots from the Hirshfeld surface analysis are shown in Fig. 5, revealing the intermolecular contacts and their percentage distributions on the Hirshfeld surface. Not surprisingly the $\mathrm{Cl} \cdots \mathrm{H} / \mathrm{H} \cdots \mathrm{Cl}$ contacts $(31.0 \%)$ are present as a major contributor, while $\mathrm{C} \cdot \mathrm{H} /$ $\mathrm{H} \cdots \mathrm{C}(14.8 \%), \mathrm{H} \cdots \mathrm{H} \quad(14.0 \%), \mathrm{O} \cdots \mathrm{H} / \mathrm{H} \cdots \mathrm{O} \quad(12.8 \%)$, $\mathrm{Cl} \cdots \mathrm{Cl} \quad(11.0 \%), \quad \mathrm{Cl} \cdots \mathrm{C} / \mathrm{C} \cdot \mathrm{Cl} \quad(8.2 \%), \quad \mathrm{Cl} \cdots \mathrm{O} / \mathrm{O} \cdots \mathrm{Cl}$ (a)

(d)

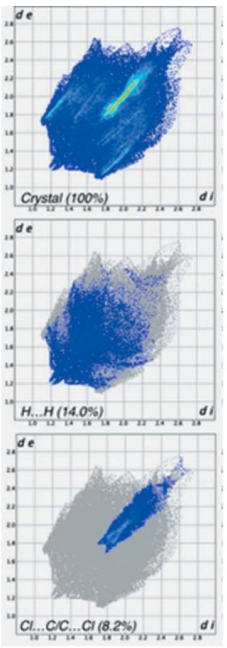

(b)

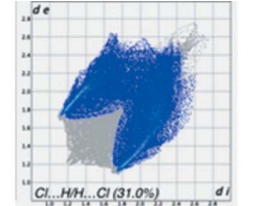

(e)

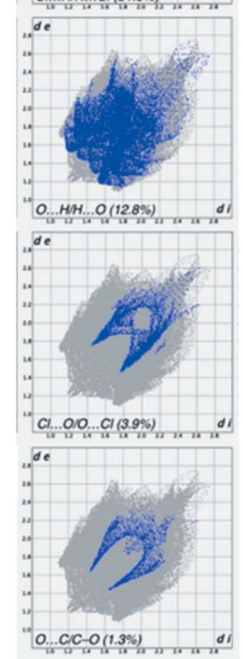

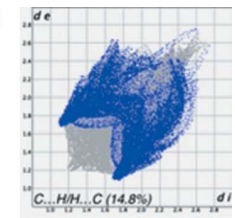

(f)

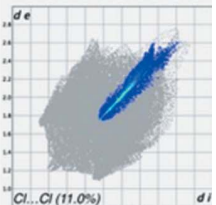

(i)

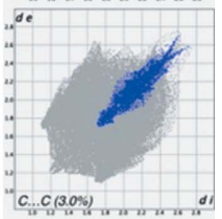

Figure 5

(a) The full two-dimensional fingerprint plot for the title compound, and those delineated into $(b) \mathrm{Cl} \cdots \mathrm{H} / \mathrm{H} \cdots \mathrm{Cl},(c) \mathrm{C} \cdots \mathrm{H} / \mathrm{H} \cdots \mathrm{C},(d) \mathrm{H} \cdots \mathrm{H},(e)$ $\mathrm{O} \cdots \mathrm{H} / \mathrm{H} \cdots \mathrm{O},(f) \mathrm{Cl} \cdots \mathrm{Cl},(g) \mathrm{Cl} \cdots \mathrm{C},(h) \mathrm{Cl} \cdots \mathrm{O}$, (i) $\mathrm{C} \cdots \mathrm{C}$ and $(j) \mathrm{O} \cdots \mathrm{C}$ contacts. 
Table 2

Experimental details.

\begin{tabular}{ll}
\hline Crystal data & \\
Chemical formula & $\mathrm{C}_{25} \mathrm{H}_{12} \mathrm{Cl}_{6} \mathrm{O}_{4}$ \\
$M_{\mathrm{r}}$ & 589.05 \\
Crystal system, space group & Monoclinic, $P 2_{1} / c$ \\
Temperature $(\mathrm{K})$ & 193 \\
$a, b, c(\AA)$ & $17.9667(4), 7.9150(1), 17.6995(5)$ \\
$\beta\left({ }^{\circ}\right)$ & $110.673(1)$ \\
$V\left(\AA^{3}\right)$ & $2354.91(9)$ \\
$Z$ & 4 \\
Radiation type & $\mathrm{Cu} K \alpha$ \\
$\mu\left(\mathrm{mm}^{-1}\right)$ & 6.95 \\
Crystal size (mm) & $0.40 \times 0.40 \times 0.20$ \\
& \\
Data collection & Rigaku R-AXIS RAPID \\
Diffractometer & Numerical $(N U M A B S ;$ Higashi, \\
Absorption correction & $1999)$ \\
& $0.168,0.337$ \\
$T_{\text {min }}, T_{\text {max }}$ & $41619,4300,3737$ \\
No. of measured, independent and & \\
$\quad$ observed $[I>2 \sigma(I)]$ reflections & 0.110 \\
$R_{\text {int }}$ & 0.602 \\
(sin $\theta / \lambda)_{\text {max }}\left(\AA^{-1}\right)$ & \\
Refinement & $0.045,0.126,1.06$ \\
$R\left[F^{2}>2 \sigma\left(F^{2}\right)\right], w R\left(F^{2}\right), S$ & 4300 \\
No. of reflections & 319 \\
No. of parameters & $\mathrm{H}-$-atom parameters constrained \\
H-atom treatment & $0.50,-0.26$ \\
$\Delta \rho_{\text {max }}, \Delta \rho_{\text {min }}\left(\mathrm{e} \AA^{-3}\right)$ &
\end{tabular}

Computer programs: PROCESS-AUTO (Rigaku, 1998), SIR2004 (Burla et al., 2007), SHELXL97 (Sheldrick, 2008) and ORTEPIII (Burnett \& Johnson, 1996).

$(3.9 \%), \mathrm{C} \cdots \mathrm{C}(3.0 \%)$ and $\mathrm{O} \cdots \mathrm{C} / \mathrm{C} \cdots \mathrm{O}(1.3 \%)$ contacts also make significant contributions to the Hirshfeld surface.

\section{Database survey}

A search of the Cambridge Structural Database (CSD, version 5.40 , last update November 2018; Groom et al., 2016) of the 2hydroxy-1-benzoylnaphthalene moiety of the title compound yield 16 hits. These include compounds with a similar aroylnaphthalene unit and other polycyclic aromatic hydrocarbon moieties (CSD refcode ITUXOM: Ji et al., 2016; PIRLUX: Freeman et al., 1994; VUDFAC: Luo \& Yu, 2009; VUDFEG: Luo \& Yu, 2009). A search with a 1-benzoyl group bonded to the 2-hydroxynaphthalene framework gave 12 hits. Among these, three had bromo group(s) at the 8-position, 3,8-positions, and 3,6-positions of the naphthalene ring core, viz. PUKGIM (Mitsui, Nakaema, Nagasawa et al., 2010), YUNWOP (Mitsui, Watanabe et al., 2010) and YUPWEM (Mitsui, Nagasawa, Watanabe et al., 2010). Four compounds had an 8-benzoyl group, i.e., 1,8-diaroylated naphthalene compounds, viz. CIQBUB (Mohri et al., 2013), LESLOM (Hijikata et al., 2013), YUQBOC (Mitsui, Nagasawa, Noguchi et al., 2010) and YUQBOC1 (Okamoto, Mitsui et al., 2012). The remaining five compounds have a single 1-benzoyl-2-hydroxynaphthalene moiety, viz. KABJUU (Nagasawa, Mitsui, Kato et al., 2010), UCUHAE (Okamoto et al., 2014), VABBEH (Nagasawa, Mitsui, Okamoto et al., 2010), VOJFOQ (Mitsui et al., 2008) and VOJFQ01 (Okamoto, Mitsui et al., 2012). These structures have $p$-substituted or unsubstituted benzoyl group(s). The structure most similar to the title compound is 1,8-bis(4-chlorobenzoyl)-7-methoxynaphthalen-2-ol ethanol solvate, for which there are two reports; refcodes YUQBOC and YUQBOC01.

\section{Synthesis and crystallization}

To a $10 \mathrm{ml}$ eggplant flask equipped with a nitrogen bulb, 2,4,6trichlorobenzoyl chloride $(0.0938 \mathrm{ml}, 0.6 \mathrm{mmol})$, dichloromethane $(0.5 \mathrm{ml})$, titanium tetrachloride $(0.1972 \mathrm{ml}$, $1.8 \mathrm{mmol}$ ), and finally 2,7-dimethoxynaphthalene $(37.6 \mathrm{mg}$, $0.2 \mathrm{mmol}$ ) were introduced sequentially. The reaction mixture was stirred at ambient temperature for $6 \mathrm{~h}$, then it was poured into ice-water. The resulting mixture was extracted with chloroform $(3 \times 20 \mathrm{ml})$, then the organic layer was washed with saturated aqueous $\mathrm{NaCl}$ solution $(3 \times 20 \mathrm{ml})$ and dried over granular $\mathrm{MgSO}_{4}$. The solvent was removed by evaporation to yield a crude product of purple viscous liquid, which was crystallized from hot (hexane $/ \mathrm{CHCl}_{3}$ ) to give yellow platelike crystals (isolated yield 24\%; m.p. 493-497 K).

${ }^{1} \mathrm{H}$ NMR $\delta\left(300 \mathrm{MHz}, \mathrm{DMSO}-d_{6}\right) ; 3.59(3 \mathrm{H}, \mathrm{s}), 7.01(1 \mathrm{H}, d, J$ $=8.4 \mathrm{~Hz}), 7.34(1 \mathrm{H}, d, J=9.3 \mathrm{~Hz}), 7.66(2 \mathrm{H}, s), 7.70(2 \mathrm{H}, s)$, $8.04(1 \mathrm{H}, d, J=8.7 \mathrm{~Hz}), 8.09(1 \mathrm{H}, d, J=9.0 \mathrm{~Hz}) \mathrm{ppm}$.

${ }^{1} \mathrm{H}$ NMR $\delta\left(300 \mathrm{MHz}, \mathrm{CDCl}_{3}\right) ; 3.45(3 \mathrm{H}, s), 6.95(1 \mathrm{H}, b r)$, $7.02(1 \mathrm{H}, d, J=8.7 \mathrm{~Hz}), 7.20(2 \mathrm{H}, b r), 7.21(1 H, \mathrm{~d}, J=9.0 \mathrm{~Hz})$, $7.42(1 \mathrm{H}, b r), 7.90(1 \mathrm{H}, d, J=8.4 \mathrm{~Hz}), 8.01(1 \mathrm{H}, d, J=9.0 \mathrm{~Hz})$ ppm.

${ }^{13} \mathrm{C} \mathrm{NMR} \delta\left(100 \mathrm{MHz}, \mathrm{CDCl}_{3}\right) ; 56.64,110.35,114.82,117.67$, $119.13,119.38,124.45,127.37,133.42,133.68,134.14,135.42$, 135.51, 136.69, 136.80, 139.15, 140.88, 165.60, 166.21, 185.98, $191.21 \mathrm{ppm}$.

IR $(\mathrm{KBr}) ; 1629(\mathrm{C}=\mathrm{O}), 1600,1510,1442$ (Ar, naphthalene), $1289(=\mathrm{C}-\mathrm{O}-\mathrm{C}) \mathrm{cm}^{-1}$.

\section{Refinement}

Crystal data, data collection and structure refinement details are summarized in Table 2. All of the $\mathrm{H}$ atoms were found in a difference-Fourier map and were subsequently refined as riding atoms, with $\mathrm{C}-\mathrm{H}=0.95$ (aromatic) and 0.96 (methyl) $\AA$, and with $U_{\text {iso }}(\mathrm{H})=1.2 U_{\text {eq }}(\mathrm{C})$.

\section{Funding information}

This work was partially supported by a Tokyo Ohka Foundation for The Promotion of Science and Technology Research Promotion Grant.

\section{References}

Burla, M. C., Caliandro, R., Camalli, M., Carrozzini, B., Cascarano, G. L., De Caro, L., Giacovazzo, C., Polidori, G., Siliqi, D. \& Spagna, R. (2007). J. Appl. Cryst. 40, 609-613.

Burnett, M. N. \& \&Johnson, C. K. (1996). ORTEPIII. Report ORNL6895. Oak Ridge National Laboratory. Tennessee, USA.

Choy, P. Y. \& Kwong, F. Y. (2013). Org. Lett. 15, 270-273.

Freeman, D., Frolow, F., Kapinus, E., Lavie, D., Lavie, G., Meruelo, D. \& Mazur, Y. (1994). J. Chem. Soc. Chem. Commun. pp. 891-892. 
Groom, C. R., Bruno, I. J., Lightfoot, M. P. \& Ward, S. C. (2016). Acta Cryst. B72, 171-179.

Higashi, T. (1999). NUMABS. Rigaku Corporation, Tokyo, Japan.

Hijikata, D., Sasagawa, K., Yoshiwaka, S., Okamoto, A. \& Yonezawa, N. (2013). Acta Cryst. E69, o208-o209.

Ji, K., Yang, F., Gao, S., Tang, J.-J. \& Gao, J. (2016). Chem. Eur. J. 22, 10225-10229.

Lee, H. \& Yi, C. S. (2015). Eur. J. Org. Chem. pp. 1899-1904.

Luo, N. \& Yu, Z. (2009). J. Organomet. Chem. 694, 3058-3067.

McKinnon, J. J., Jayatilaka, D. \& Spackman, M. A. (2007). Chem. Commun. pp. 3814-3816.

Mitsui, R., Nagasawa, A., Noguchi, K., Okamoto, A. \& Yonezawa, N. (2010). Acta Cryst. E66, o1790.

Mitsui, R., Nagasawa, A., Watanabe, S., Okamoto, A. \& Yonezawa, N. (2010). Acta Cryst. E66, o1761.

Mitsui, R., Nakaema, K., Nagasawa, A., Noguchi, K. \& Yonezawa, N. (2010). Acta Cryst. E66, o676.

Mitsui, R., Nakaema, K., Noguchi, K. \& Yonezawa, N. (2008). Acta Cryst. E64, o2497.

Mitsui, R., Watanabe, S., Nagasawa, A., Okamoto, A. \& Yonezawa, N. (2010). Acta Cryst. E66, o1304.

Mohri, S., Yoshiwaka, S., Isozaki, K., Yonezawa, N. \& Okamoto, A. (2013). Acta Cryst. C69, 1541-1544.

Murashige, R., Hayashi, Y., Ohmori, S., Torii, A., Aizu, Y., Muto, Y., Murai, Y., Oda, Y. \& Hashimoto, M. (2011). Tetrahedron, 67, 641649.

Muto, T., Sasagawa, K., Okamoto, A., Oike, H. \& Yonezawa, N. (2012a). Acta Cryst. E68, o23.

Muto, T., Sasagawa, K., Okamoto, A., Oike, H. \& Yonezawa, N. (2012b). Acta Cryst. E68, o906.

Naeimi, H., Amini, A. \& Moradian, M. (2014). Org. Chem. Front. 1, 415-421.

Nagasawa, A., Mitsui, R., Kato, Y., Okamoto, A. \& Yonezawa, N. (2010). Acta Cryst. E66, o2677.

Nagasawa, A., Mitsui, R., Okamoto, A. \& Yonezawa, N. (2010). Acta Cryst. E66, o2820-02821.
Nakaema, K., Okamoto, A., Noguchi, K. \& Yonezawa, N. (2007). Acta Cryst. E63, o4120.

Nimnual, P., Tummatorn, J., Thongsornkleeb, C. \& Ruchirawat, S. (2015). J. Org. Chem. 80, 8657-8667.

Nonhebel, D. C. (1968). Tetrahedron, 24, 1869-1874.

Ohisa, S., Saeki, M., Shiomichi, H., Yonezawa, N. \& Okamoto, A. (2018). Eur. Chem. Bull. 7, 1-9.

Okamoto, A., Mitsui, R., Oike, H. \& Yonezawa, N. (2011). Chem. Lett. 40, 1283-1284.

Okamoto, A., Mitsui, R., Watanabe, S., Tsubouchi, T. \& Yonezawa, N. (2012). Int. J. Org. Chem. 02, 194-201.

Okamoto, A., Nagasawa, A. \& Yonezawa, N. (2014). Eur. Chem. Bull. 3, 263-268.

Okamoto, A., Watanabe, S., Nakaema, K. \& Yonezawa, N. (2012). Cryst. Struc. Theo. Appl. 1, 121-127.

Okamoto, A. \& Yonezawa, N. (2009). Chem. Lett. 38, 914-915.

Okamoto, A. \& Yonezawa, N. (2015). J. Syn. Org. Chem. Jpn. 73, 339360.

Rigaku (1998). PROCESS-AUTO. Rigaku Corporation, Tokyo, Japan.

Schiel, C., Oelgemoller, M. \& Mattay, J. (2001). Synthesis, pp. 12751279.

Sheldrick, G. M. (2008). Acta Cryst. A64, 112-122.

Siskos, M. G., Tzakos, A. G. \& Gerothanassis, I. P. (2015). Org. Biomol. Chem. 13, 8852-8868.

Spackman, M. A. \& Jayatilaka, D. (2009). CrystEngComm, 11, 19-32.

Tran, P. H., Phung, H. Q., Duong, M. N. \& Pham-Tran, N.-N. (2017). Tetrahedron Lett. 58, 1588-1563.

Turner, M. J., McKinnon, J. J., Wolff, S. K., Grimwood, D. J., Spackman, P. R., Jayatilaka, D. \& Spackman, M. A. (2017). CrystalExplorer17. University of Western Australia. http://hirshfeldsurface.net

Weng, F., Wang, C. \& Xu, B. (2010). Tetrahedron Lett. 51, 2593-2596.

Yoshiwaka, S., Ogata, K., Yonezawa, N. \& Okamoto, A. (2015). Eur. Chem. Bull. 4(4), 195-201.

Zhou, C. \& Larock, R. C. (2004). J. Am. Chem. Soc. 126, 2302-2303. 


\section{supporting information}

Acta Cryst. (2019). E75, 1418-1422［https://doi.org/10.1107/S2056989019012118]

Crystal structure and Hirshfeld surface analysis of 2-hydroxy-7-methoxy-1,8-bis(2,4,6-trichlorobenzoyl)naphthalene

\section{Toyokazu Muto, Kikuko lida, Keiichi Noguchi, Noriyuki Yonezawa and Akiko Okamoto}

Computing details

Data collection: PROCESS-AUTO (Rigaku, 1998); cell refinement: PROCESS-AUTO (Rigaku, 1998); data reduction:

PROCESS-AUTO (Rigaku, 1998); program(s) used to solve structure: SIR2004 (Burla et al., 2007); program(s) used to refine structure: SHELXL97 (Sheldrick, 2008); molecular graphics: ORTEPIII (Burnett \& Johnson, 1996); software used to prepare material for publication: SHELXL97 (Sheldrick, 2008).

7-Methoxy-1,8-bis[(2,4,6-trichlorophenyl)carbonyl]naphthalen-2-ol

Crystal data

$\mathrm{C}_{25} \mathrm{H}_{12} \mathrm{Cl}_{6} \mathrm{O}_{4}$

$M_{r}=589.05$

Monoclinic, $P 2_{1} / c$

Hall symbol: -P 2ybc

$a=17.9667$ (4) $\AA$

$b=7.9150(1) \AA$

$c=17.6995(5) \AA$

$\beta=110.673(1)^{\circ}$

$V=2354.91(9) \AA^{3}$

$Z=4$

\section{Data collection}

Rigaku R-AXIS RAPID

diffractometer

Radiation source: rotaing anode

Graphite monochromator

Detector resolution: 10.000 pixels $\mathrm{mm}^{-1}$

$\omega$ scans

Absorption correction: numerical

(NUMABS; Higashi, 1999)

$T_{\min }=0.168, T_{\max }=0.337$

Refinement

Refinement on $F^{2}$

Least-squares matrix: full

$R\left[F^{2}>2 \sigma\left(F^{2}\right)\right]=0.045$

$w R\left(F^{2}\right)=0.126$

$S=1.06$

4300 reflections

319 parameters

0 restraints
$F(000)=1184$

$D_{\mathrm{x}}=1.661 \mathrm{Mg} \mathrm{m}^{-3}$

$\mathrm{Cu} K \alpha$ radiation, $\lambda=1.54187 \AA$

Cell parameters from 28116 reflections

$\theta=3.0-68.3^{\circ}$

$\mu=6.95 \mathrm{~mm}^{-1}$

$T=193 \mathrm{~K}$

Platelet, yellow

$0.40 \times 0.40 \times 0.20 \mathrm{~mm}$

41619 measured reflections

4300 independent reflections

3737 reflections with $I>2 \sigma(I)$

$R_{\text {int }}=0.110$

$\theta_{\max }=68.3^{\circ}, \theta_{\min }=5.1^{\circ}$

$h=-21 \rightarrow 21$

$k=-9 \rightarrow 9$

$l=-21 \rightarrow 21$

Primary atom site location: structure-invariant direct methods

Secondary atom site location: difference Fourier map

Hydrogen site location: inferred from neighbouring sites

$\mathrm{H}$-atom parameters constrained

$w=1 /\left[\sigma^{2}\left(F_{\mathrm{o}}^{2}\right)+(0.0625 P)^{2}+1.5934 P\right]$

where $P=\left(F_{\mathrm{o}}^{2}+2 F_{\mathrm{c}}^{2}\right) / 3$ 
$(\Delta / \sigma)_{\max }<0.001$

$\Delta \rho_{\max }=0.50 \mathrm{e} \AA^{-3}$

$\Delta \rho_{\min }=-0.26 \mathrm{e} \AA^{-3}$
Extinction correction: SHELXL97 (Sheldrick, 2008), $\mathrm{Fc}^{*}=\mathrm{kFc}\left[1+0.001 \mathrm{xFc}^{2} \lambda^{3} / \sin (2 \theta)\right]^{-1 / 4}$

Extinction coefficient: $0.00166(17)$

\section{Special details}

Geometry. All esds (except the esd in the dihedral angle between two 1.s. planes) are estimated using the full covariance matrix. The cell esds are taken into account individually in the estimation of esds in distances, angles and torsion angles; correlations between esds in cell parameters are only used when they are defined by crystal symmetry. An approximate (isotropic) treatment of cell esds is used for estimating esds involving l.s. planes.

Refinement. Refinement of $\mathrm{F}^{2}$ against ALL reflections. The weighted R-factor $\mathrm{wR}$ and goodness of fit $\mathrm{S}$ are based on $\mathrm{F}^{2}$, conventional R-factors $R$ are based on $F$, with $F$ set to zero for negative $\mathrm{F}^{2}$. The threshold expression of $\mathrm{F}^{2}>2 \operatorname{sigma}\left(\mathrm{F}^{2}\right)$ is used only for calculating R-factors(gt) etc. and is not relevant to the choice of reflections for refinement. R-factors based on $\mathrm{F}^{2}$ are statistically about twice as large as those based on F, and R- factors based on ALL data will be even larger.

Fractional atomic coordinates and isotropic or equivalent isotropic displacement parameters $\left(\hat{A}^{2}\right)$

\begin{tabular}{|c|c|c|c|c|}
\hline & $x$ & $y$ & $z$ & $U_{\text {iso }} * / U_{\text {eq }}$ \\
\hline $\mathrm{Cl1}$ & $0.13592(4)$ & $0.62787(10)$ & $0.05084(5)$ & $0.0543(2)$ \\
\hline $\mathrm{Cl} 2$ & $0.00708(5)$ & $0.06020(13)$ & $0.10904(5)$ & 0.0645 \\
\hline $\mathrm{Cl3}$ & $0.32240(4)$ & $0.09099(9)$ & $0.20159(4)$ & $0.0477(2)$ \\
\hline $\mathrm{Cl} 4$ & $0.09824(5)$ & $0.59970(12)$ & $0.24340(5)$ & $0.0634(3)$ \\
\hline $\mathrm{Cl} 5$ & $0.10530(6)$ & $1.19664(13)$ & $0.39062(6)$ & $0.0703(3)$ \\
\hline $\mathrm{Cl} 6$ & $0.37761(4)$ & $0.90853(10)$ & $0.39693(4)$ & $0.0512(2)$ \\
\hline $\mathrm{O} 1$ & $0.30235(12)$ & $0.5154(3)$ & 0.07105 (12) & $0.0517(5)$ \\
\hline $\mathrm{O} 2$ & $0.26606(11)$ & $0.6928(3)$ & $0.20713(11)$ & $0.0422(5)$ \\
\hline $\mathrm{O} 3$ & $0.44648(13)$ & $0.4173(3)$ & $0.11247(14)$ & $0.0589(6)$ \\
\hline $\mathrm{H} 3 \mathrm{~A}$ & 0.4035 & 0.4583 & 0.0812 & $0.071^{*}$ \\
\hline $\mathrm{O} 4$ & $0.26290(15)$ & $0.5184(3)$ & $0.41656(12)$ & $0.0613(6)$ \\
\hline $\mathrm{C} 1$ & $0.37736(16)$ & $0.4494(4)$ & $0.20717(17)$ & $0.0413(6)$ \\
\hline $\mathrm{C} 2$ & $0.44482(17)$ & $0.4097(4)$ & $0.1885(2)$ & $0.0492(7)$ \\
\hline $\mathrm{C} 3$ & $0.51384(17)$ & $0.3460(4)$ & $0.2480(2)$ & $0.0571(9)$ \\
\hline $\mathrm{H} 3$ & 0.5593 & 0.3195 & 0.2347 & $0.069 *$ \\
\hline $\mathrm{C} 4$ & $0.51548(18)$ & $0.3226(4)$ & $0.3237(2)$ & $0.0584(9)$ \\
\hline $\mathrm{H} 4$ & 0.5612 & 0.2728 & 0.3625 & $0.070 *$ \\
\hline $\mathrm{C} 5$ & $0.45068(18)$ & $0.3702(4)$ & 0.34747 (19) & $0.0509(8)$ \\
\hline C6 & $0.4532(2)$ & $0.3417(4)$ & 0.42678 (19) & $0.0604(9)$ \\
\hline H6 & 0.4992 & 0.2905 & 0.4644 & $0.072 *$ \\
\hline $\mathrm{C} 7$ & $0.3920(2)$ & $0.3850(4)$ & $0.45164(19)$ & $0.0583(9)$ \\
\hline $\mathrm{H} 7$ & 0.3938 & 0.3581 & 0.5046 & $0.070^{*}$ \\
\hline $\mathrm{C} 8$ & $0.3262(2)$ & $0.4701(4)$ & $0.39744(17)$ & $0.0511(8)$ \\
\hline $\mathrm{C} 9$ & $0.32242(16)$ & $0.5103(4)$ & $0.31878(16)$ & $0.0409(6)$ \\
\hline $\mathrm{C} 10$ & $0.38174(16)$ & $0.4428(4)$ & 0.28935 (17) & $0.0418(6)$ \\
\hline $\mathrm{C} 11$ & $0.30243(16)$ & 0.4589 (4) & $0.13587(16)$ & $0.0403(6)$ \\
\hline $\mathrm{C} 12$ & $0.22876(15)$ & $0.3656(4)$ & $0.13489(15)$ & $0.0379(6)$ \\
\hline $\mathrm{C} 13$ & $0.15151(17)$ & $0.4292(4)$ & $0.09543(16)$ & $0.0431(7)$ \\
\hline C14 & $0.08428(16)$ & $0.3378(4)$ & $0.08818(16)$ & $0.0464(7)$ \\
\hline H14 & 0.0331 & 0.3860 & 0.0625 & $0.056^{*}$ \\
\hline C15 & $0.09164(16)$ & $0.1760(4)$ & $0.11843(17)$ & $0.0458(7)$ \\
\hline C16 & $0.16576(17)$ & 0.1018 & $0.15470(16)$ & $0.0457(7)$ \\
\hline
\end{tabular}




$\begin{array}{lllll}\text { H16 } & 0.1706 & -0.0115 & 0.1736 & 0.055^{*} \\ \text { C17 } & 0.23220(15) & 0.1976(4) & 0.16237(15) & 0.0386(6) \\ \text { C18 } & 0.27267(15) & 0.6515(4) & 0.27587(16) & 0.0395(6) \\ \text { C19 } & 0.23318(16) & 0.7726(4) & 0.31692(15) & 0.0398(6) \\ \text { C20 } & 0.15062(16) & 0.7704(4) & 0.29719(16) & 0.0452(7) \\ \text { C21 } & 0.11036(18) & 0.8971(4) & 0.31971(18) & 0.0500(7) \\ \text { H21 } & 0.0543 & 0.8925 & 0.3057 & 0.060^{*} \\ \text { C22 } & 0.15427(18) & 1.0321(4) & 0.36357(18) & 0.0503(7) \\ \text { C23 } & 0.23617(18) & 1.0364(4) & 0.38688(17) & 0.0480(7) \\ \text { H23 } & 0.2656 & 1.1279 & 0.4180 & 0.058^{*} \\ \text { C24 } & 0.27431(16) & 0.9059(4) & 0.36436(16) & 0.0424(6) \\ \text { C25 } & 0.2684(3) & 0.5207(6) & 0.4997(2) & 0.0862(14) \\ \text { H25A } & 0.2202 & 0.5722 & 0.5036 & 0.103^{*} \\ \text { H25B } & 0.3150 & 0.5868 & 0.5317 & 0.103^{*} \\ \text { H25C } & 0.2736 & 0.4048 & 0.5205 & 0.103^{*} \\ \end{array}$

Atomic displacement parameters $\left(\AA^{2}\right)$

\begin{tabular}{|c|c|c|c|c|c|c|}
\hline & $U^{11}$ & $U^{22}$ & $U^{33}$ & $U^{12}$ & $U^{13}$ & $U^{23}$ \\
\hline $\mathrm{Cl1}$ & $0.0419(4)$ & $0.0540(5)$ & $0.0572(4)$ & $0.0039(3)$ & $0.0054(3)$ & $0.0116(3)$ \\
\hline $\mathrm{Cl} 2$ & $0.0421(4)$ & $0.0769(6)$ & $0.0739(5)$ & $-0.0167(4)$ & 0.0198 (4) & 0.0057 (4) \\
\hline $\mathrm{Cl} 3$ & $0.0375(4)$ & $0.0437(4)$ & $0.0543(4)$ & $0.0019(3)$ & $0.0067(3)$ & $0.0027(3)$ \\
\hline $\mathrm{Cl} 4$ & $0.0434(4)$ & $0.0766(6)$ & $0.0739(5)$ & $-0.0202(4)$ & $0.0255(4)$ & $-0.0219(4)$ \\
\hline $\mathrm{Cl} 5$ & $0.0680(6)$ & $0.0673(6)$ & $0.0806(6)$ & $0.0184(5)$ & $0.0324(5)$ & $-0.0061(4)$ \\
\hline $\mathrm{Cl} 6$ & $0.0357(4)$ & $0.0568(5)$ & $0.0525(4)$ & $-0.0032(3)$ & 0.0049 (3) & $-0.0037(3)$ \\
\hline $\mathrm{O} 1$ & $0.0434(11)$ & $0.0682(15)$ & $0.0466(11)$ & $-0.0015(10)$ & $0.0198(9)$ & $0.0071(10)$ \\
\hline $\mathrm{O} 2$ & $0.0418(10)$ & $0.0444(11)$ & $0.0401(10)$ & $0.0018(9)$ & $0.0139(8)$ & $0.0033(8)$ \\
\hline $\mathrm{O} 3$ & $0.0461(12)$ & $0.0644(16)$ & $0.0731(15)$ & $0.0009(11)$ & $0.0297(11)$ & $-0.0043(12)$ \\
\hline $\mathrm{O} 4$ & $0.0764(16)$ & $0.0679(16)$ & $0.0452(12)$ & $-0.0017(13)$ & $0.0284(11)$ & $0.0088(11)$ \\
\hline $\mathrm{C} 1$ & $0.0307(13)$ & $0.0372(15)$ & $0.0531(16)$ & $-0.0021(11)$ & $0.0111(12)$ & $-0.0008(12)$ \\
\hline $\mathrm{C} 2$ & $0.0356(15)$ & $0.0438(18)$ & $0.0666(19)$ & $-0.0069(13)$ & $0.0161(14)$ & $-0.0040(14)$ \\
\hline $\mathrm{C} 3$ & $0.0312(15)$ & $0.0483(19)$ & $0.086(2)$ & $-0.0005(13)$ & $0.0137(15)$ & $-0.0100(17)$ \\
\hline $\mathrm{C} 4$ & $0.0330(15)$ & $0.0430(18)$ & $0.079(2)$ & $0.0006(13)$ & $-0.0053(15)$ & $-0.0020(16)$ \\
\hline $\mathrm{C} 5$ & $0.0391(16)$ & $0.0430(18)$ & $0.0542(17)$ & $-0.0043(13)$ & $-0.0037(13)$ & $-0.0053(13)$ \\
\hline C6 & $0.057(2)$ & $0.0471(19)$ & $0.0503(18)$ & $-0.0023(16)$ & $-0.0145(15)$ & $0.0031(14)$ \\
\hline $\mathrm{C} 7$ & $0.069(2)$ & $0.052(2)$ & $0.0395(16)$ & -0.0097 (17) & $0.0013(15)$ & $0.0044(14)$ \\
\hline $\mathrm{C} 8$ & 0.0587 (19) & $0.0451(18)$ & $0.0425(15)$ & $-0.0083(15)$ & $0.0093(14)$ & $0.0011(13)$ \\
\hline C9 & $0.0370(14)$ & $0.0414(16)$ & $0.0382(14)$ & $-0.0050(12)$ & $0.0057(11)$ & $0.0009(12)$ \\
\hline $\mathrm{C} 10$ & $0.0332(14)$ & $0.0337(15)$ & $0.0497(15)$ & $-0.0041(11)$ & $0.0036(11)$ & $0.0001(12)$ \\
\hline $\mathrm{C} 11$ & $0.0361(14)$ & $0.0421(16)$ & $0.0414(14)$ & $0.0020(12)$ & $0.0123(11)$ & $-0.0003(12)$ \\
\hline $\mathrm{C} 12$ & $0.0336(13)$ & $0.0445(16)$ & $0.0332(13)$ & $-0.0010(12)$ & $0.0090(10)$ & $-0.0010(11)$ \\
\hline $\mathrm{C} 13$ & $0.0384(14)$ & $0.0486(17)$ & $0.0371(14)$ & $0.0000(13)$ & $0.0068(11)$ & $0.0021(12)$ \\
\hline $\mathrm{C} 14$ & $0.0324(14)$ & $0.061(2)$ & $0.0421(15)$ & $0.0021(13)$ & $0.0085(11)$ & $-0.0004(13)$ \\
\hline $\mathrm{C} 15$ & $0.0350(14)$ & $0.059(2)$ & $0.0423(15)$ & $-0.0096(13)$ & $0.0124(11)$ & $-0.0020(13)$ \\
\hline $\mathrm{C} 16$ & $0.0418(16)$ & $0.0502(19)$ & $0.0429(15)$ & $-0.0053(13)$ & $0.0121(12)$ & $0.0032(13)$ \\
\hline $\mathrm{C} 17$ & $0.0329(13)$ & $0.0451(16)$ & $0.0351(13)$ & $0.0006(12)$ & $0.0088(10)$ & $-0.0021(11)$ \\
\hline $\mathrm{C} 18$ & $0.0312(13)$ & $0.0440(16)$ & $0.0398(14)$ & $-0.0047(12)$ & $0.0080(11)$ & $0.0000(12)$ \\
\hline C19 & $0.0358(13)$ & $0.0473(17)$ & $0.0367(13)$ & $-0.0022(12)$ & $0.0132(11)$ & $0.0027(12)$ \\
\hline
\end{tabular}




\begin{tabular}{lllllll}
\hline & & & & & \\
$\mathrm{C} 20$ & $0.0366(14)$ & $0.0576(19)$ & $0.0413(14)$ & $-0.0087(13)$ & $0.0138(11)$ & $-0.0023(13)$ \\
$\mathrm{C} 21$ & $0.0383(15)$ & $0.067(2)$ & $0.0480(16)$ & $0.0024(14)$ & $0.0186(13)$ & $0.0005(15)$ \\
$\mathrm{C} 22$ & $0.0510(17)$ & $0.055(2)$ & $0.0489(16)$ & $0.0111(15)$ & $0.0225(14)$ & $0.0034(14)$ \\
$\mathrm{C} 23$ & $0.0494(17)$ & $0.0501(18)$ & $0.0421(15)$ & $0.0006(14)$ & $0.0132(13)$ & $-0.0025(13)$ \\
$\mathrm{C} 24$ & $0.0339(14)$ & $0.0523(18)$ & $0.0383(14)$ & $-0.0010(12)$ & $0.0096(11)$ & $0.0015(12)$ \\
$\mathrm{C} 25$ & $0.127(4)$ & $0.090(3)$ & $0.049(2)$ & $-0.016(3)$ & $0.040(2)$ & $-0.003(2)$ \\
\hline
\end{tabular}

Geometric parameters $\left(\AA,{ }^{o}\right)$

\begin{tabular}{|c|c|c|c|}
\hline $\mathrm{C} 11-\mathrm{C} 13$ & $1.737(3)$ & $\mathrm{C} 7-\mathrm{H} 7$ & 0.9500 \\
\hline $\mathrm{C} 12-\mathrm{C} 15$ & $1.731(3)$ & $\mathrm{C} 8-\mathrm{C} 9$ & $1.406(4)$ \\
\hline $\mathrm{Cl} 3-\mathrm{C} 17$ & $1.740(3)$ & $\mathrm{C} 9-\mathrm{C} 10$ & $1.444(4)$ \\
\hline $\mathrm{C} 14-\mathrm{C} 20$ & $1.728(3)$ & $\mathrm{C} 9-\mathrm{C} 18$ & $1.465(4)$ \\
\hline $\mathrm{C} 15-\mathrm{C} 22$ & $1.731(3)$ & $\mathrm{C} 11-\mathrm{C} 12$ & $1.511(4)$ \\
\hline $\mathrm{C} 16-\mathrm{C} 24$ & $1.738(3)$ & $\mathrm{C} 12-\mathrm{C} 13$ & $1.408(4)$ \\
\hline $\mathrm{O} 1-\mathrm{C} 11$ & $1.231(3)$ & $\mathrm{C} 12-\mathrm{C} 17$ & 1.409 (4) \\
\hline $\mathrm{O} 2-\mathrm{C} 18$ & $1.224(3)$ & $\mathrm{C} 13-\mathrm{C} 14$ & $1.374(4)$ \\
\hline $\mathrm{O} 3-\mathrm{C} 2$ & $1.358(4)$ & $\mathrm{C} 14-\mathrm{C} 15$ & $1.376(5)$ \\
\hline $\mathrm{O} 3-\mathrm{H} 3 \mathrm{~A}$ & 0.8400 & $\mathrm{C} 14-\mathrm{H} 14$ & 0.9500 \\
\hline $\mathrm{O} 4-\mathrm{C} 8$ & $1.351(4)$ & $\mathrm{C} 15-\mathrm{C} 16$ & $1.388(4)$ \\
\hline $\mathrm{O} 4-\mathrm{C} 25$ & $1.441(4)$ & $\mathrm{C} 16-\mathrm{C} 17$ & $1.380(4)$ \\
\hline $\mathrm{C} 1-\mathrm{C} 2$ & $1.400(4)$ & $\mathrm{C} 16-\mathrm{H} 16$ & 0.9500 \\
\hline $\mathrm{C} 1-\mathrm{C} 10$ & $1.430(4)$ & $\mathrm{C} 18-\mathrm{C} 19$ & $1.522(4)$ \\
\hline $\mathrm{C} 1-\mathrm{C} 11$ & $1.487(4)$ & $\mathrm{C} 19-\mathrm{C} 24$ & $1.388(4)$ \\
\hline $\mathrm{C} 2-\mathrm{C} 3$ & $1.407(4)$ & $\mathrm{C} 19-\mathrm{C} 20$ & $1.399(4)$ \\
\hline $\mathrm{C} 3-\mathrm{C} 4$ & $1.342(5)$ & $\mathrm{C} 20-\mathrm{C} 21$ & $1.375(4)$ \\
\hline $\mathrm{C} 3-\mathrm{H} 3$ & 0.9500 & $\mathrm{C} 21-\mathrm{C} 22$ & $1.391(5)$ \\
\hline $\mathrm{C} 4-\mathrm{C} 5$ & $1.421(5)$ & $\mathrm{C} 21-\mathrm{H} 21$ & 0.9500 \\
\hline $\mathrm{C} 4-\mathrm{H} 4$ & 0.9500 & $\mathrm{C} 22-\mathrm{C} 23$ & $1.381(4)$ \\
\hline $\mathrm{C} 5-\mathrm{C} 6$ & $1.407(5)$ & $\mathrm{C} 23-\mathrm{C} 24$ & $1.374(4)$ \\
\hline $\mathrm{C} 5-\mathrm{C} 10$ & $1.422(4)$ & $\mathrm{C} 23-\mathrm{H} 23$ & 0.9500 \\
\hline $\mathrm{C} 6-\mathrm{C} 7$ & $1.365(5)$ & $\mathrm{C} 25-\mathrm{H} 25 \mathrm{~A}$ & 0.9800 \\
\hline C6- 6 6 & 0.9500 & $\mathrm{C} 25-\mathrm{H} 25 \mathrm{~B}$ & 0.9800 \\
\hline $\mathrm{C} 7-\mathrm{C} 8$ & $1.403(5)$ & $\mathrm{C} 25-\mathrm{H} 25 \mathrm{C}$ & 0.9800 \\
\hline $\mathrm{C} 2-\mathrm{O} 3-\mathrm{H} 3 \mathrm{~A}$ & 109.5 & $\mathrm{C} 12-\mathrm{C} 13-\mathrm{C} 11$ & $121.4(2)$ \\
\hline $\mathrm{C} 8-\mathrm{O} 4-\mathrm{C} 25$ & $120.0(3)$ & $\mathrm{C} 13-\mathrm{C} 14-\mathrm{C} 15$ & $119.5(3)$ \\
\hline $\mathrm{C} 2-\mathrm{C} 1-\mathrm{C} 10$ & $119.4(3)$ & $\mathrm{C} 13-\mathrm{C} 14-\mathrm{H} 14$ & 120.2 \\
\hline $\mathrm{C} 2-\mathrm{C} 1-\mathrm{C} 11$ & $114.3(3)$ & $\mathrm{C} 15-\mathrm{C} 14-\mathrm{H} 14$ & 120.2 \\
\hline $\mathrm{C} 10-\mathrm{C} 1-\mathrm{C} 11$ & $125.0(2)$ & $\mathrm{C} 14-\mathrm{C} 15-\mathrm{C} 16$ & $121.2(3)$ \\
\hline $\mathrm{O} 3-\mathrm{C} 2-\mathrm{C} 1$ & $123.2(3)$ & $\mathrm{C} 14-\mathrm{C} 15-\mathrm{Cl} 2$ & $119.6(2)$ \\
\hline $\mathrm{O} 3-\mathrm{C} 2-\mathrm{C} 3$ & $115.8(3)$ & $\mathrm{C} 16-\mathrm{C} 15-\mathrm{Cl} 2$ & $119.1(3)$ \\
\hline $\mathrm{C} 1-\mathrm{C} 2-\mathrm{C} 3$ & $120.8(3)$ & $\mathrm{C} 17-\mathrm{C} 16-\mathrm{C} 15$ & $117.9(3)$ \\
\hline $\mathrm{C} 4-\mathrm{C} 3-\mathrm{C} 2$ & $120.0(3)$ & $\mathrm{C} 17-\mathrm{C} 16-\mathrm{H} 16$ & 121.0 \\
\hline $\mathrm{C} 4-\mathrm{C} 3-\mathrm{H} 3$ & 120.0 & $\mathrm{C} 15-\mathrm{C} 16-\mathrm{H} 16$ & 121.0 \\
\hline $\mathrm{C} 2-\mathrm{C} 3-\mathrm{H} 3$ & 120.0 & $\mathrm{C} 16-\mathrm{C} 17-\mathrm{C} 12$ & $123.6(3)$ \\
\hline $\mathrm{C} 3-\mathrm{C} 4-\mathrm{C} 5$ & $121.8(3)$ & $\mathrm{C} 16-\mathrm{C} 17-\mathrm{Cl} 3$ & $115.1(2)$ \\
\hline $\mathrm{C} 3-\mathrm{C} 4-\mathrm{H} 4$ & 119.1 & $\mathrm{C} 12-\mathrm{C} 17-\mathrm{Cl} 3$ & $121.1(2)$ \\
\hline
\end{tabular}




\begin{tabular}{|c|c|}
\hline $\mathrm{C} 5-\mathrm{C} 4-\mathrm{H} 4$ & 119.1 \\
\hline $\mathrm{C} 6-\mathrm{C} 5-\mathrm{C} 4$ & $120.7(3)$ \\
\hline $\mathrm{C} 6-\mathrm{C} 5-\mathrm{C} 10$ & $120.0(3)$ \\
\hline $\mathrm{C} 4-\mathrm{C} 5-\mathrm{C} 10$ & $119.2(3)$ \\
\hline $\mathrm{C} 7-\mathrm{C} 6-\mathrm{C} 5$ & $122.4(3)$ \\
\hline $\mathrm{C} 7-\mathrm{C} 6-\mathrm{H} 6$ & 118.8 \\
\hline $\mathrm{C} 5-\mathrm{C} 6-\mathrm{H} 6$ & 118.8 \\
\hline $\mathrm{C} 6-\mathrm{C} 7-\mathrm{C} 8$ & $118.7(3)$ \\
\hline $\mathrm{C} 6-\mathrm{C} 7-\mathrm{H} 7$ & 120.7 \\
\hline $\mathrm{C} 8-\mathrm{C} 7-\mathrm{H} 7$ & 120.7 \\
\hline $\mathrm{O} 4-\mathrm{C} 8-\mathrm{C} 7$ & $123.0(3)$ \\
\hline $\mathrm{O} 4-\mathrm{C} 8-\mathrm{C} 9$ & $115.7(3)$ \\
\hline $\mathrm{C} 7-\mathrm{C} 8-\mathrm{C} 9$ & $121.3(3)$ \\
\hline $\mathrm{C} 8-\mathrm{C} 9-\mathrm{C} 10$ & $119.3(3)$ \\
\hline $\mathrm{C} 8-\mathrm{C} 9-\mathrm{C} 18$ & $119.6(3)$ \\
\hline $\mathrm{C} 10-\mathrm{C} 9-\mathrm{C} 18$ & $119.2(2)$ \\
\hline $\mathrm{C} 5-\mathrm{C} 10-\mathrm{C} 1$ & $118.1(3)$ \\
\hline $\mathrm{C} 5-\mathrm{C} 10-\mathrm{C} 9$ & $117.0(3)$ \\
\hline $\mathrm{C} 1-\mathrm{C} 10-\mathrm{C} 9$ & $124.8(2)$ \\
\hline $\mathrm{O} 1-\mathrm{C} 11-\mathrm{C} 1$ & $120.8(3)$ \\
\hline $\mathrm{O} 1-\mathrm{C} 11-\mathrm{C} 12$ & $117.0(2)$ \\
\hline $\mathrm{C} 1-\mathrm{C} 11-\mathrm{C} 12$ & $120.8(2)$ \\
\hline $\mathrm{C} 13-\mathrm{C} 12-\mathrm{C} 17$ & $115.1(3)$ \\
\hline $\mathrm{C} 13-\mathrm{C} 12-\mathrm{C} 11$ & $122.5(3)$ \\
\hline $\mathrm{C} 17-\mathrm{C} 12-\mathrm{C} 11$ & $121.7(2)$ \\
\hline $\mathrm{C} 14-\mathrm{C} 13-\mathrm{C} 12$ & $122.6(3)$ \\
\hline $\mathrm{C} 14-\mathrm{C} 13-\mathrm{C} 11$ & $116.0(2)$ \\
\hline $\mathrm{C} 10-\mathrm{C} 1-\mathrm{C} 2-\mathrm{O} 3$ & $-178.1(3)$ \\
\hline $\mathrm{C} 11-\mathrm{C} 1-\mathrm{C} 2-\mathrm{O} 3$ & $14.5(4)$ \\
\hline $\mathrm{C} 10-\mathrm{C} 1-\mathrm{C} 2-\mathrm{C} 3$ & $7.0(4)$ \\
\hline $\mathrm{C} 11-\mathrm{C} 1-\mathrm{C} 2-\mathrm{C} 3$ & $-160.4(3)$ \\
\hline $\mathrm{O} 3-\mathrm{C} 2-\mathrm{C} 3-\mathrm{C} 4$ & $-175.3(3)$ \\
\hline $\mathrm{C} 1-\mathrm{C} 2-\mathrm{C} 3-\mathrm{C} 4$ & $0.0(5)$ \\
\hline $\mathrm{C} 2-\mathrm{C} 3-\mathrm{C} 4-\mathrm{C} 5$ & $-3.9(5)$ \\
\hline $\mathrm{C} 3-\mathrm{C} 4-\mathrm{C} 5-\mathrm{C} 6$ & $178.9(3)$ \\
\hline $\mathrm{C} 3-\mathrm{C} 4-\mathrm{C} 5-\mathrm{C} 10$ & $0.8(5)$ \\
\hline $\mathrm{C} 4-\mathrm{C} 5-\mathrm{C} 6-\mathrm{C} 7$ & $-179.7(3)$ \\
\hline $\mathrm{C} 10-\mathrm{C} 5-\mathrm{C} 6-\mathrm{C} 7$ & $-1.6(5)$ \\
\hline $\mathrm{C} 5-\mathrm{C} 6-\mathrm{C} 7-\mathrm{C} 8$ & $-3.9(5)$ \\
\hline $\mathrm{C} 25-\mathrm{O} 4-\mathrm{C} 8-\mathrm{C} 7$ & $15.0(5)$ \\
\hline $\mathrm{C} 25-\mathrm{O} 4-\mathrm{C} 8-\mathrm{C} 9$ & $-165.1(3)$ \\
\hline $\mathrm{C} 6-\mathrm{C} 7-\mathrm{C} 8-\mathrm{O} 4$ & $-179.8(3)$ \\
\hline $\mathrm{C} 6-\mathrm{C} 7-\mathrm{C} 8-\mathrm{C} 9$ & $0.2(5)$ \\
\hline $\mathrm{O} 4-\mathrm{C} 8-\mathrm{C} 9-\mathrm{C} 10$ & $-171.3(3)$ \\
\hline $\mathrm{C} 7-\mathrm{C} 8-\mathrm{C} 9-\mathrm{C} 10$ & $8.7(4)$ \\
\hline $\mathrm{O} 4-\mathrm{C} 8-\mathrm{C} 9-\mathrm{C} 18$ & $24.5(4)$ \\
\hline $\mathrm{C} 7-\mathrm{C} 8-\mathrm{C} 9-\mathrm{C} 18$ & $-155.6(3)$ \\
\hline
\end{tabular}

\begin{tabular}{|c|c|}
\hline $\mathrm{O} 2-\mathrm{C} 18-\mathrm{C} 9$ & $123.1(3)$ \\
\hline $\mathrm{O} 2-\mathrm{C} 18-\mathrm{C} 19$ & $114.1(2)$ \\
\hline $\mathrm{C} 9-\mathrm{C} 18-\mathrm{C} 19$ & $122.4(2)$ \\
\hline $\mathrm{C} 24-\mathrm{C} 19-\mathrm{C} 20$ & $116.9(3)$ \\
\hline $\mathrm{C} 24-\mathrm{C} 19-\mathrm{C} 18$ & $122.0(2)$ \\
\hline $\mathrm{C} 20-\mathrm{C} 19-\mathrm{C} 18$ & $120.3(2)$ \\
\hline $\mathrm{C} 21-\mathrm{C} 20-\mathrm{C} 19$ & $122.6(3)$ \\
\hline $\mathrm{C} 21-\mathrm{C} 20-\mathrm{C} 14$ & $119.3(2)$ \\
\hline $\mathrm{C} 19-\mathrm{C} 20-\mathrm{Cl} 4$ & $118.1(2)$ \\
\hline $\mathrm{C} 20-\mathrm{C} 21-\mathrm{C} 22$ & $118.0(3)$ \\
\hline $\mathrm{C} 20-\mathrm{C} 21-\mathrm{H} 21$ & 121.0 \\
\hline $\mathrm{C} 22-\mathrm{C} 21-\mathrm{H} 21$ & 121.0 \\
\hline $\mathrm{C} 23-\mathrm{C} 22-\mathrm{C} 21$ & $121.3(3)$ \\
\hline $\mathrm{C} 23-\mathrm{C} 22-\mathrm{C} 15$ & $119.4(3)$ \\
\hline $\mathrm{C} 21-\mathrm{C} 22-\mathrm{C} 15$ & $119.3(2)$ \\
\hline $\mathrm{C} 24-\mathrm{C} 23-\mathrm{C} 22$ & $118.9(3)$ \\
\hline $\mathrm{C} 24-\mathrm{C} 23-\mathrm{H} 23$ & 120.6 \\
\hline $\mathrm{C} 22-\mathrm{C} 23-\mathrm{H} 23$ & 120.6 \\
\hline $\mathrm{C} 23-\mathrm{C} 24-\mathrm{C} 19$ & $122.2(3)$ \\
\hline $\mathrm{C} 23-\mathrm{C} 24-\mathrm{C} 16$ & $118.6(2)$ \\
\hline $\mathrm{C} 19-\mathrm{C} 24-\mathrm{C} 16$ & $119.2(2)$ \\
\hline $\mathrm{O} 4-\mathrm{C} 25-\mathrm{H} 25 \mathrm{~A}$ & 109.5 \\
\hline $\mathrm{O} 4-\mathrm{C} 25-\mathrm{H} 25 \mathrm{~B}$ & 109.5 \\
\hline $\mathrm{H} 25 \mathrm{~A}-\mathrm{C} 25-\mathrm{H} 25 \mathrm{~B}$ & 109.5 \\
\hline $\mathrm{O} 4-\mathrm{C} 25-\mathrm{H} 25 \mathrm{C}$ & 109.5 \\
\hline $\mathrm{H} 25 \mathrm{~A}-\mathrm{C} 25-\mathrm{H} 25 \mathrm{C}$ & 109.5 \\
\hline $\mathrm{H} 25 \mathrm{~B}-\mathrm{C} 25-\mathrm{H} 25 \mathrm{C}$ & 109.5 \\
\hline $\mathrm{C} 17-\mathrm{C} 12-\mathrm{C} 13-\mathrm{C} 14$ & $3.5(4)$ \\
\hline $\mathrm{C} 11-\mathrm{C} 12-\mathrm{C} 13-\mathrm{C} 14$ & $174.0(3)$ \\
\hline $\mathrm{C} 17-\mathrm{C} 12-\mathrm{C} 13-\mathrm{Cl}$ & $-175.0(2$ \\
\hline $\mathrm{C} 11-\mathrm{C} 12-\mathrm{C} 13-\mathrm{Cl1}$ & $-4.6(4)$ \\
\hline $\mathrm{C} 12-\mathrm{C} 13-\mathrm{C} 14-\mathrm{C} 15$ & $-1.8(4)$ \\
\hline $\mathrm{C} 11-\mathrm{C} 13-\mathrm{C} 14-\mathrm{C} 15$ & $176.8(2)$ \\
\hline $\mathrm{C} 13-\mathrm{C} 14-\mathrm{C} 15-\mathrm{C} 16$ & $-1.4(4)$ \\
\hline $\mathrm{C} 13-\mathrm{C} 14-\mathrm{C} 15-\mathrm{Cl} 2$ & $-179.7(2$ \\
\hline $\mathrm{C} 14-\mathrm{C} 15-\mathrm{C} 16-\mathrm{C} 17$ & $2.5(4)$ \\
\hline $\mathrm{C} 12-\mathrm{C} 15-\mathrm{C} 16-\mathrm{C} 17$ & $-179.2(2$ \\
\hline $\mathrm{C} 15-\mathrm{C} 16-\mathrm{C} 17-\mathrm{C} 12$ & $-0.5(4)$ \\
\hline $\mathrm{C} 15-\mathrm{C} 16-\mathrm{C} 17-\mathrm{Cl} 3$ & $-175.5(2$ \\
\hline $\mathrm{C} 13-\mathrm{C} 12-\mathrm{C} 17-\mathrm{C} 16$ & $-2.3(4)$ \\
\hline $\mathrm{C} 11-\mathrm{C} 12-\mathrm{C} 17-\mathrm{C} 16$ & $-172.9(3$ \\
\hline $\mathrm{C} 13-\mathrm{C} 12-\mathrm{C} 17-\mathrm{Cl} 3$ & $172.3(2)$ \\
\hline $\mathrm{C} 11-\mathrm{C} 12-\mathrm{C} 17-\mathrm{Cl} 3$ & $1.8(4)$ \\
\hline $\mathrm{C} 8-\mathrm{C} 9-\mathrm{C} 18-\mathrm{O} 2$ & $-179.4(3$ \\
\hline $\mathrm{C} 10-\mathrm{C} 9-\mathrm{C} 18-\mathrm{O} 2$ & $16.3(4)$ \\
\hline $\mathrm{C} 8-\mathrm{C} 9-\mathrm{C} 18-\mathrm{C} 19$ & $8.5(4)$ \\
\hline $\mathrm{C} 10-\mathrm{C} 9-\mathrm{C} 18-\mathrm{C} 19$ & $-155.8(3$ \\
\hline
\end{tabular}




$\begin{array}{llll}\mathrm{C} 6-\mathrm{C} 5-\mathrm{C} 10-\mathrm{C} 1 & -172.0(3) & \mathrm{O} 2-\mathrm{C} 18-\mathrm{C} 19-\mathrm{C} 24 & -91.9(3) \\ \mathrm{C} 4-\mathrm{C} 5-\mathrm{C} 10-\mathrm{C} 1 & 6.1(4) & \mathrm{C} 9-\mathrm{C} 18-\mathrm{C} 19-\mathrm{C} 24 & 80.8(4) \\ \mathrm{C} 6-\mathrm{C} 5-\mathrm{C} 10-\mathrm{C} 9 & 10.2(4) & \mathrm{O} 2-\mathrm{C} 18-\mathrm{C} 19-\mathrm{C} 20 & 77.3(3) \\ \mathrm{C} 4-\mathrm{C} 5-\mathrm{C} 10-\mathrm{C} 9 & -171.7(3) & \mathrm{C} 9-\mathrm{C} 18-\mathrm{C} 19-\mathrm{C} 20 & -109.9(3) \\ \mathrm{C} 2-\mathrm{C} 1-\mathrm{C} 10-\mathrm{C} 5 & -9.9(4) & \mathrm{C} 24-\mathrm{C} 19-\mathrm{C} 20-\mathrm{C} 21 & 3.1(4) \\ \mathrm{C} 11-\mathrm{C} 1-\mathrm{C} 10-\mathrm{C} 5 & 156.1(3) & \mathrm{C} 18-\mathrm{C} 19-\mathrm{C} 20-\mathrm{C} 21 & -166.7(3) \\ \mathrm{C} 2-\mathrm{C} 1-\mathrm{C} 10-\mathrm{C} 9 & 167.7(3) & \mathrm{C} 24-\mathrm{C} 19-\mathrm{C} 20-\mathrm{C} 14 & -176.2(2) \\ \mathrm{C} 11-\mathrm{C} 1-\mathrm{C} 10-\mathrm{C} 9 & -26.3(4) & \mathrm{C} 18-\mathrm{C} 19-\mathrm{C} 20-\mathrm{C} 14 & 14.1(4) \\ \mathrm{C} 8-\mathrm{C} 9-\mathrm{C} 10-\mathrm{C} 5 & -13.6(4) & \mathrm{C} 19-\mathrm{C} 20-\mathrm{C} 21-\mathrm{C} 22 & 0.2(4) \\ \mathrm{C} 18-\mathrm{C} 9-\mathrm{C} 10-\mathrm{C} 5 & 150.6(3) & \mathrm{C} 14-\mathrm{C} 20-\mathrm{C} 21-\mathrm{C} 22 & 179.5(2) \\ \mathrm{C} 8-\mathrm{C} 9-\mathrm{C} 10-\mathrm{C} 1 & 168.7(3) & \mathrm{C} 20-\mathrm{C} 21-\mathrm{C} 22-\mathrm{C} 23 & -2.6(4) \\ \mathrm{C} 18-\mathrm{C} 9-\mathrm{C} 10-\mathrm{C} 1 & -27.0(4) & \mathrm{C} 20-\mathrm{C} 21-\mathrm{C} 22-\mathrm{C} 15 & 178.3(2) \\ \mathrm{C} 2-\mathrm{C} 1-\mathrm{C} 11-\mathrm{O} 1 & -36.1(4) & \mathrm{C} 21-\mathrm{C} 22-\mathrm{C} 23-\mathrm{C} 24 & 1.6(4) \\ \mathrm{C} 10-\mathrm{C} 1-\mathrm{C} 11-\mathrm{O} 1 & 157.3(3) & \mathrm{C} 15-\mathrm{C} 22-\mathrm{C} 23-\mathrm{C} 24 & -179.3(2) \\ \mathrm{C} 2-\mathrm{C} 1-\mathrm{C} 11-\mathrm{C} 12 & 130.2(3) & \mathrm{C} 22-\mathrm{C} 23-\mathrm{C} 24-\mathrm{C} 19 & 2.0(4) \\ \mathrm{C} 10-\mathrm{C} 1-\mathrm{C} 11-\mathrm{C} 12 & -36.4(4) & \mathrm{C} 22-\mathrm{C} 23-\mathrm{C} 24-\mathrm{C} 16 & -177.8(2) \\ \mathrm{O} 1-\mathrm{C} 11-\mathrm{C} 12-\mathrm{C} 13 & -47.0(4) & \mathrm{C} 20-\mathrm{C} 19-\mathrm{C} 24-\mathrm{C} 23 & -4.2(4) \\ \mathrm{C} 1-\mathrm{C} 11-\mathrm{C} 12-\mathrm{C} 13 & 146.3(3) & \mathrm{C} 18-\mathrm{C} 19-\mathrm{C} 24-\mathrm{C} 23 & 165.4(3) \\ \mathrm{O} 1-\mathrm{C} 11-\mathrm{C} 12-\mathrm{C} 17 & 122.9(3) & \mathrm{C} 20-\mathrm{C} 19-\mathrm{C} 24-\mathrm{C} 16 & 175.5(2) \\ \mathrm{C} 1-\mathrm{C} 11-\mathrm{C} 12-\mathrm{C} 17 & -43.9(4) & \mathrm{C} 18-\mathrm{C} 19-\mathrm{C} 24-\mathrm{C} 16 & -14.9(4) \\ \end{array}$

Hydrogen-bond geometry $\left(A,{ }^{\circ}\right)$

\begin{tabular}{lllll}
\hline$D-\mathrm{H} \cdots A$ & $D-\mathrm{H}$ & $\mathrm{H} \cdots A$ & $D \cdots A$ & $D-\mathrm{H} \cdots A$ \\
\hline $\mathrm{O} 3-\mathrm{H} 3 A \cdots \mathrm{O} 1$ & 0.84 & 1.82 & $2.551(3)$ & 145 \\
\hline
\end{tabular}

